\title{
TRANSFERENCIA. INTEGRACIÓN DEL PSICOANÁLISIS Y LA LOGOTERAPIA
}

\section{Transfer. Psychoanalysis Integration and Logotherapy}

Patricia Ovejas*

\section{Resumen}

El presente artículo analiza la integración de los aportes del Psicoanálisis, la Logoterapia y la Filosofía en el ámbito de la clínica. Se llevó a cabo una exhaustiva revisión bibliográfica adoptando una perspectiva crítica, y como fuentes primarias, se analizaron las obras de Freud, Frankl, diversos filósofos y autores psicoanalistas y existencialistas. Las principales conclusiones son que a partir de una determinada idea de hombre y la profundización del concepto de transferencia, se revela que la integración entre el Psicoanálisis, la Logoterapia y Filosofía no sólo es posible sino sumamente necesaria; por otra parte el hombre, múltiple en su esencia, necesita de abordajes múltiples e integradores para alcanzar la salud mental.

Palabras clave: Idea de hombre, Transferencia, Psicoanálisis, Logoterapia, Filosofía.

\begin{abstract}
The present article analyzes the integration of Psychoanalysis contributions, Logotherapy and Philosophy in the clinical area. An exhaustive bibliographical review was carried out adopting a critical perspective, and as primary sources, Freud, Frankl, several philosophers and psychoanalyst authors as well as existentialists' works were analized. The main conclusions are that since a particular idea of a man and going towards the transfer concept, it is revealed that the integration among Psychoanalysis, Logotherapy and Philosophy is not only possible but vitally necessary. On the other hand, the man, so different in their essence, will need multiple approaches and integrators to reach mental health.
\end{abstract}

Key words: The idea of the man, Transfer, Psychoanalysis, Logotherapy, Philosophy.

* Psicóloga. Docente de la Pontificia Universidad Católica Santa María de Buenos Aires. patriciaovejas@yahoo.com.ar 


\section{DEFINICIÓN Y CLARIFICACIÓN DEL PROBLEMA}

El principal problema que presentan las distintas escuelas psicológicas es el reduccionismo: toman un aspecto -parcial- del hombre y lo convierten en la totalidad. La Logoterapia, en cambio, no incurre en este error, al presentarse como un enfoque abarcativo e integrador, complementario a los otros enfoques.

Ustedes recuerdan que Frankl dice que la logoterapia no es una panacea, que es una terapia específica en algunos casos, como en las neurosis noógenas. En otros casos es terapia de complementación (Oro, 1985, p. 12).

Todas las escuelas psicológicas tienen una determinada idea del hombre y una determinada idea del mundo, aunque generalmente ni explicitada ni reconocida.

Freud parte de una idea reduccionista del hombre basada en la teoría de Darwin -que plantea una diferencia sólo de grado entre el hombre y los mamíferos superiores-. Si bien Freud sacó a la Medicina de una visión estrictamente biologista, no se cuestionó la idea del mundo que se desprende de su teoría, aunque sí dejó en claro que habría que preguntarse sobre las neurosis también desde el ámbito de la Filosofía. Ya en A propósito de un caso de neurosis obsesiva ("El hombre de las ratas"), Freud expresa que el paciente distorsiona su idea filosófica del universo.

Los biologistas se centran solamente en la dimensión biológica. Por lo tanto, consideran que las enfermedades requieren de una terapéutica eminentemente farmacológica. Esta corriente tiene mayor fundamento dentro del terreno de las psicosis, en especial en la esquizofrenia y la psicosis maníacodepresiva, y las epilepsias. Freud hace hincapié en la profundidad inconsciente. Trata al paciente desde lo más profundo de su dimensión psicológica utilizando, para el diagnóstico y el tratamiento, el trabajo con la transferencia. Pero Freud no fue más allá de la problemática del psicoanálisis donde todo es causación y donde se quiere justificar toda la psicología a través de una fundamentación biológica, porque no se propuso otra cosa: él quiso dar lugar al instinto que no era reconocido en la época victoriana.

Viktor Frankl, en cambio, completa esta idea de hombre de Freud, sosteniendo que el hombre es siempre libre para elegir, cimentando su antropología en Santo Tomás de Aquino -que define al hombre como unidad en la multiplicidad- y en el aporte de Teilhard de Chardin -que distingue al hombre del animal por una diferencia de especie, por su ser espiritual y por su inventiva puesta al servicio de superar los obstáculos de la vida-. El hombre es una unidad bio-psicoespiritual y debe ser atendido en cada una de sus dimensiones. Frankl destaca la jerarquía de lo espiritual que permite el manejo de lo biológico y lo psicológico. Para Frankl, lo espiritual no es un epifenómeno sino algo superior, y en esto coincide con Nicolai Hartmann.

Nicolai Hartmann (el creador de la Teoría de los Estratos), en la cuidadosa investigación que ha hecho sobre las Ciencias de la Naturaleza y las Ciencias de la Cultura, afirma que los estratos inferiores no producen a los superiores, es decir, que el espíritu no es sublimación de lo instintivo, sino que es algo distinto. Si bien, los estratos inferiores son los más fuertes, éstos son menos libres que el estrato superior. La libertad recién aparece en el estrato espiritual. De la fuerza de lo inferior, provienen los condicionamientos que la logoterapia no ignora. De la libertad, proviene lo que Frankl denomina la capacidad de oposición del espíritu.

La aparición del estrato espiritual se da por un sistema de complejidad creciente. Lo espiritual no es un elemento aislado sino que es lo que estructura a la totalidad y le otorga dinamismo. En la dimensión biológica hay pulsiones, necesidades; lo espiritual es lo que permite el manejo de las mismas. Por ello, el tratamiento de esta dimensión superior hace más eficiente el trabajo psicoterapéutico, al afianzar, completar y confirmar el tratamiento de las otras dimensiones. Si no se trabaja con lo espiritual, el paciente vuelve a enfermar. Por ello, Frankl llama a la Logoterapia una psicoterapia de complementación, mientras las restantes escuelas se proponen como autosuficientes, capaces de diagnosticar y curar todo desde su enfoque. Aquí radica el valioso aporte de Frankl, que no considera al hombre en forma fragmentaria sino como una totalidad, una unidad bio- 
psico-espiritual, al que hay que abordar psicoterapéuticamente desde cada estrato y con técnicas específicas para cada estrato. Cuando la patología neurótica es grave se necesita un abordaje profundo y completo desde las tres dimensiones: biológica Psicofarmacología-, psicológica -Psicoanálisis-, y espiritual -Logoterapia-.

Cuando las escuelas psicológicas se quieren trasladar como totalidades, no cumplen su función. No obstante, se pueden tomar los elementos que son útiles de cada una de ellas e integrarlos en un tratamiento junto con los aportes de la Medicina, la Psiquiatría y la Filosofía. No pueden ignorarse los aportes del psicoanálisis al campo de la salud mental. En la clínica, es indispensable trabajar a partir de los mecanismos de defensa, la explicación de las enfermedades desde las series complementarias y el abordaje de la transferencia. Sin embargo, frente a la subversión de los valores que plantea la postmodernidad, es obligación de los psicólogos dar una respuesta eficiente. Por lo tanto, será necesario que los profesionales de la salud mental estén capacitados para trabajar, también desde el psicoanálisis, con la problemática filosófica que traen los pacientes.

\section{SÍNTESIS DE INVESTIGACIONES PREVIAS}

Entre fines del siglo XIX y principios del XX, Sigmund Freud demuestra una preocupación que resulta innovadora. En forma paulatina, se independiza de la tradición descriptiva buscando la causalidad y consolidando un salto cualitativo en el conocimiento de la enfermedad mental. En el siglo XX, la ciencia, que se había independizado de la religión, se erige como un pilar fundamental. La palabra adviene en concepto, se denomina representación, adquiere solidez. Ha transcurrido más de un siglo desde que Freud presentó los primeros enunciados sobre la neurosis. Los ideales modernos se refieren -por su fundamento positivista-, a la separación de la ciencia y la fe y de la razón y la filosofía clásica. La neurosis es la patología del siglo $\mathrm{XX}$, y su estudio está inmerso en este proceso. Por ello, no se han integrado, hasta ahora, los aportes del psicoanálisis y la filosofía en el tratamiento de las mismas.
Freud, utilizando el método positivista -propio de la ciencia en su época-, se interesa en las neurosis actuales y las psiconeurosis, y descubre que la sexualidad es un factor determinante y causal. Éste es el signo que distingue su aporte: la importancia capital de la sexualidad, en la etiología de las neurosis. Freud retrocede, en la búsqueda de la causalidad de las neurosis, hasta la sexualidad en la infancia. Este elemento causal insoslayable introdujo una innovación que se encuentra en la categoría de la temporalidad, relacionando lo actual con lo pasado. Categorización particular del tiempo que sigue siendo, hasta hoy, uno de los aportes distintivos de la teoría freudiana. Cuando se profundiza en el concepto de transferencia, queda claro que las consecuencias que la sexualidad produjo repercuten y modifican la concepción lineal de la temporalidad. Desde entonces, los sucesos del pasado se encuentran en el presente, revestidos de una vigencia actual. El niño y la infancia adquieren un nuevo valor; introduce un cambio radical adjudicándole sexualidad a la vida infantil. Freud, en los primeros años, de regreso de La Salpetrière, se aboca a la Pediatría, ejerciendo influencia entre sus adeptos con sus teorías. Freud tenía la convicción de que los problemas sexuales ocupaban los pensamientos de todo niño antes de llegar a la pubertad. Asimismo, es posible ver a Freud en la vanguardia de las rupturas epistemológicas fundamentales del siglo al introducir la noción de inconsciente, quitando centralidad al yo en la determinación de las acciones. El yo deja de ser el centro de la escena psíquica.

El trabajo terapéutico, propuesto por Freud en sus comienzos, consistía en desarrollar una labor que contrarrestara la fuerza psíquica opuesta por el paciente a recordar las representaciones patógenas que habían sido excluidas del tráfico asociativo de la conciencia. Freud deduce que el enfermo se libera de los síntomas histéricos en cuanto reproduce las impresiones patógenas causales, dándoles expresión verbal y exteriorizando el afecto concomitante. La imposibilidad de descarga hará que el recuerdo se convierta en patógeno. En la búsqueda de lo oculto, conceptualizó la hipótesis del inconsciente, encontrando allí, las ideas acerca de la realidad psíquica y la vida fantasmática, que se hallan a un paso del complejo de Edipo. 
El complejo de Edipo se inicia en la fase fálica de la evolución de la libido. Esta denominación aparece en La organización genital infantil (1923), en la que la premisa universal del falo ya ha sido reconocida como teoría sexual infantil. Dicho complejo se constituye como nódulo de las neurosis.

Paulatinamente, Freud fue desarrollando el gran aporte del psicoanálisis a la clínica de las neurosis, que es la implementación del tratamiento a partir del trabajo con la transferencia. En Sobre la dinámica de la transferencia (1912) afirmaba que la misma:

... surge necesariamente en toda cura psicoanalítica (...) nuestras investigaciones nos han revelado que sólo una parte de las tendencias que determinan la vida erótica han realizado una evolución psíquica completa (...) una parte de las tendencias ha quedado detenida en su desarrollo o ha quedado confinada en lo inconsciente (...) el individuo orientará estas representaciones libidinosas hacia toda nueva persona que surja en su horizonte... (Freud, 1991, p.1468)

Por ello, las condiciones del psicoanálisis establecidas por Freud desde el principio, implican reglas imperativas -comparadas por Freud a las reglas de la asepsia para los cirujanos-. El analista, es definido a menudo como una pantalla blanca un espejo, y estas expresiones ponen en evidencia el carácter de neutralidad de su presencia. El analista es un interlocutor, que en su discreción, debe experimentar y comprender al mismo tiempo que el paciente, lo que él experimenta, es decir, más de lo que él expresa. Espejo e interlocutor, el analista es también al que se le representa en todo instante el conjunto de la situación.

Esta relación será el verdadero tema y lo que se juega en el análisis, y el paciente se defiende frente del analista, por los mismos medios que emplea el neurótico para defenderse contra sus pulsiones.

Durante el curso del psicoanálisis, las situaciones de insatisfacción del sujeto tienden a reproducirse y a repetirse de una manera estereotipada en la relación viviente entre él y el analista. ...es, por lo tanto perfectamente normal y comprensible que la carga de libido que el individuo insatisfecho mantiene esperanzadamente, pronto se oriente también hacia la persona del médico (...) esta carga se atendrá a ciertos modelos (...) el modelo de esta inclusión habría de ser correspondiente a la imagen del padre (...) pero la transferencia no tiene que seguir obligadamente este prototipo y puede establecerse también conforme a la imagen de la madre o del hermano... (Freud, 1991, p. 1469)

Poco a poco toda la situación neurótica se desplaza así sobre la situación analítica, frustrante por sí misma puesto que el sujeto no recibe nada que responda a sus exigencias neuróticas.

El fenómeno de la transferencia es un fenómeno de la vida psíquica de valor absolutamente general: el amor y el odio son las manifestaciones más típicas. El análisis permite comprenderlas, pero solamente la transferencia en el interior de la relación analista-analizado es susceptible de una utilización técnica. En efecto, las condiciones operatorias del análisis y, por consecuencia, la seguridad de su método, se limitan al campo definido por la relación fundamental (Ey, 1996, p.1027).

Y esta relación fundamental no debe llevarse a cabo de cualquier modo:

El analista que procede en la línea de la analítica de la existencia que él propugna, no podrá seguir a Freud en la aplicación que éste hace de la transferencia. Sino que más bien el terapeuta de la línea del análisis de la existencia hace valer el amor de transferencia como la auténtica relación humana (Frankl, 1974, p. 314).

Todas las psicoterapias hacen uso de la transferencia -sabiéndolo o no, incluso los que la niegan-, pero sus condiciones técnicas no permiten un verdadero estudio de la situación transferencial. El psicoanálisis, en cambio, basa su eficacia en esta relación transferencial, por lo que en él encontramos su desarrollo y sus fundamentos. La transferencia es evidentemente una experiencia que no se desarrolla 
sólo en el paciente, ya que un fenómeno simétrico se produce en el analista: la contra-transferencia.

Las neurosis pueden ser tratadas de diversas maneras, pero hay una sola forma de entenderlas en sus dinamismos inconscientes, causales: psicoanalíticamente, es decir, en lo más profundo de sus mecanismos. Simmel reclamó que todo psicoterapeuta, sin importar cuál sea el método que se propusiera aplicar, tuviera una formación psicoanalítica completa (Ovejas, 2005).

Gran parte de los ataques dirigidos a los psicoanalistas se basan en la idea de que éstos giran siempre alrededor de sus propias premisas. Pero más allá de las críticas, hay que aceptar que el aporte del psicoanálisis en el entendimiento de la génesis de la enfermedad mental es un conocimiento ineludible a la hora del abordaje terapéutico. Sin embargo, se debe tener presente que el abordaje psicoanalítico no es el punto donde termina el tratamiento: es el método desde el cual se diagnóstica y se comienza el tratamiento, para partir desde allí a la integración con los aportes de la Logoterapia.

Cuanto más cerca se halle una neurosis del extremo traumático de la conocida serie complementaria, mayores serán las posibilidades de éxito de los esfuerzos externos de apoyo. Si el paciente alcanza a ver las relaciones existentes entre síntomas, preocupaciones o partes de su personalidad hasta ese momento inconexas, si se le ayuda a descubrir los patrones generales de conducta detrás de cada acto concreto, todo ello dará a su yo, por cierto, una mayor fortaleza. No obstante, cabe aclarar, que este fortalecimiento puede ser usado, en ocasiones, perjudicialmente, en beneficio de la resistencia.

Diversos autores sostienen que una ayuda racional de esta índole ya es psicoanálisis. En realidad, constituye el primer paso en los tratamientos psicoanalíticos, el cual no consiste en descifrar patrones de conducta, sino en producir una alteración dinámica en las condiciones de las cuales surgieron dichos patrones patológicos.

La base de toda psiconeurosis es el conflicto neurótico. Únicamente una alteración de las relaciones dinámicas de los elementos constituyentes de este conflicto puede alterar la neurosis. La relación afectiva del paciente con el analista, en su transferencia, altera la relación dinámica entre impulsos y angustia.

Una neurosis de transferencia es el sustituto más frecuente e importante de una neurosis espontánea. Lo que Freud quiso significar con la expresión «establecimiento de una neurosis de transferencia» era que los conflictos instintivos infantiles reprimidos hallan su expresión en la relación afectiva con el analista, y por ello ya no tienen necesidad de ninguna otra expresión, o por lo menos, no necesitan tantas expresiones como antes.

Ante la pregunta sobre cómo opera el psicoanálisis profano, es decir, cómo operan todos aquellos métodos de psicoterapia que no van tan lejos como Freud en este aspecto, es posible responder que pueden hacerlo de diferentes maneras, pero si logran algún éxito, puede deberse a una combinación de los mecanismos siguientes: (a) mejorías de transferencia alcanzadas mediante cambios dinámicos debidos a la relación afectiva con el analista; (b) ayuda racional mediante la verbalización de sus dificultades reales; (c) una ayuda inespecífica consistente en dirigir la atención del paciente hacia asuntos que hasta ese momento no había considerado. No obstante, si la neurosis es grave, una cura sencilla de este tipo se hace imposible.

Por otro lado, la psicoterapia, a menudo, es concebida como una reeducación. No obstante, la misma sólo será posible si se trabaja a partir y a través de la relación transferencial. Hablar de repetición en Freud implica desarrollar el concepto de rememoración. Para él, rememorar era volver a recordar a fin de que los síntomas desaparecieran por el devenir consciente. Freud consideraba el síntoma como producción en lugar del recuerdo. La rememoración sería un volver a recordar que da lugar al olvido por la desaparición de lo reprimido. Pero ello presenta dificultades. La paciente histérica rememora hasta la última nota sin que por ello haya curación. En el obsesivo la rememoración es causa de anulación y aislamiento. Hay fracaso en la rememoración; en este punto de fracaso, de ignorancia, va a surgir la repetición. Freud afirma: "El analizado no recuerda nada de lo reprimido (...) sino que lo vive de nuevo. 
No lo reproduce como recuerdo, si no como acto" (Ovejas, 1992, p. 1). Por lo tanto, la repetición es una forma de recordar como puesta en acto. Dicha puesta cobra la forma de una resistencia contra la aparición del recuerdo que traerá aparejada una agravación de los síntomas en la cura. Los síntomas adquieren así una nueva significación: la neurosis de transferencia. Por lo tanto, la repetición como puesta en acto del lazo pasado olvidado es: la transferencia. Freud la definía diciendo:

la transferencia no es por sí misma más que una repetición, la transferencia del pretérito olvidado, pero no sólo sobre el médico sino sobre todos los sectores de la situación presente (...) no debemos tratarla como un hecho histórico, sino como una potencia actual (Freud, 1991, p. 1685)

La transferencia no es por sí misma más que una repetición, pero no sólo sobre el psicólogo, sino sobre todos los demás sectores de la situación presente. El analizado sustituye el impulso a recordar no sólo en lo que afecta a su relación con el analista sino también en todas las demás actividades y relaciones simultáneas de su vida. La transferencia crea así una zona intermedia entre la enfermedad y la vida y, a través de ella, va teniendo efecto la transición desde la primera a la segunda. El manejo temporal de la transferencia constituye el principal recurso para frenar el automatismo de repetición y transformarlo en razón para recordar.

El analista, sostiene Freud en Construcciones en el análisis (1937), tiene que construir, que interpretar. Así logrará la convicción del paciente sin que haya rememoración. Pero muchas veces esto sólo no alcanza. La repetición guarda una relación fundamental con lo perdido.

En Más allá del principio de placer (1920) Freud reformula en otros términos la relación de la repetición con la pulsión de muerte. La insatisfacción aparece así en el principio de la repetición:

... es indudable que la repetición constituye una fuente de placer. En cambio, en el analizado se ve claramente que la obsesión de repetir en la transferencia los sucesos de la infancia se opone en absoluto al principio de placer. El enfermo se conduce en estos casos por completo infantilmente y nos muestra, de ese modo, que las reprimidas huellas mnémicas de sus experiencias primeras no se hallan en él en estado de ligadura ni son hasta cierto punto capaces de procesos secundarios (Ovejas, 1992, p. 3).

Quedan definidas dos compulsiones de repetición: una pura como lo no-ligado de experiencias dolorosas y traumáticas, la otra atraída hacia el principio de placer y permitiendo la búsqueda de lo perdido. Por lo tanto, en la transferencia la compulsión a la repetición es para Freud consecuencia de un clivaje del yo.

Las interrupciones en la cura llevan a Freud a pensar que la repetición es, entonces, recuerdo de un fracaso, de un malogramiento y que no tiene que ver con el placer como principio. Las pulsiones sexuales no ligadas, para Freud, vienen a trastornar el equilibrio económico del aparato psíquico, y la compulsión a la repetición cumple, para él, una función de ligazón concerniente a esas pulsiones sexuales no ligadas con la pulsión de muerte.

En El problema económico del masoquismo (1924), Freud introduce el masoquismo originario como punto de transición entre el principio de nirvana (tendencia a la pulsión de muerte) y el principio de placer. Aquí, encuentra el anudamiento entre la pulsión de vida y la pulsión de muerte. La experiencia traumática es entendida como fuente de excitación: el displacer como objeto de placer. Los padecimientos que la neurosis trae consigo constituyen precisamente el factor que da a esta enfermedad un alto valor para la tendencia masoquista. Resulta muy instructivo comprobar que una neurosis que ha desafiado todos los esfuerzos terapéuticos puede desaparecer, contra todos los principios teóricos, una vez que el sujeto contrae un matrimonio que lo hace desgraciado, pierde su fortuna o contrae una peligrosa enfermedad orgánica. Un padecimiento queda entonces sustituido por otro y puede comprobarse que de lo que se trataba era tan sólo de poder conservar cierta medida de dolor.

La repetición sintomática debe ser articulada con el beneficio primario del síntoma en relación con la insatisfacción y trabajar a partir de ello. De esta manera, 
se abre un trabajo fundamental del analista a partir de la repetición.

\section{IDENTIFICACIÓN DE RELACIONES}

Cuando hay lazo, hay transferencia con el otro que escucha, hay demanda de amor al otro y tiempo de cierre del inconsciente. En ese momento de báscula transferencial, el analista debe eclipsarse haciendo desaparecer su yo. El analista, presta su yo al otro en ese momento de intensa transferencia, y el sujeto recibe su mensaje. Tiempo de apertura del inconsciente, la realidad de la transferencia es también presencia del pasado, pero presencia en un acto que es creación, que es oportunidad para aprehender lo no aprehendido; se repite para lograr lo aún no logrado. A partir de este concepto debe intervenir el analista. Pero además de la lectura psicoanalítica no podemos dejar de leer este concepto también desde la Logoterapia, que ve en cada situación una oportunidad para el crecimiento del sujeto, para la búsqueda de un sentido que lo lleve a crecer, que lo conduzca a la felicidad, que proviene del logro de una mayor perfección, la cual se logra, también, a partir de la superación de los obstáculos.

El hecho de nombrar contenidos inconscientes, que no están aún representados por derivados preconscientes y que por ello no pueden ser reconocidos como tales por el paciente, por el sólo hecho de dirigir hacia ellos su atención, no es interpretar. Ello se produce haciendo uso de la transferencia positiva y de identificaciones transitorias con el analista.

La atmósfera analítica, que convence al paciente de que nada tiene que temer en el hecho de tolerar impulsos anteriormente rechazados, parece ser no solamente un prerrequisito de toda interpretación de la transferencia sino un elemento fundamental en el trabajo con ella. El peligro es convertir el análisis en una especie de análisis teórico que habla del pasado sin notar que está aún presente. El análisis deberá demostrar la persistencia efectiva del pasado en el presente.

Este hecho que se hace, en lo posible, en la instancia transferencial, es lo que distingue al psicoanálisis de todas las otras psicoterapias.
Todos los métodos psicoterapéuticos hacen uso de la transferencia, pero sólo en el psicoanálisis este uso consiste en una interpretación de la transferencia, es decir, en hacerla consciente (Fenichel, 1996, p. 635).

El hecho de que los conflictos patógenos revividos en la transferencia son sentidos en el presente con todo su contenido emocional, es lo que hace que la eficacia de interpretación de la transferencia exceda tanto a la de cualquier otra interpretación. Las partes rechazadas de los instintos han conservado su carácter infantil solamente porque fueron rechazadas. Si la defensa es anulada, aquello que fue excluido recobra su conexión con la personalidad madura y se acomoda a ella. La sexualidad pregenital rechazada ha estado resistiendo a la primacía genital. Una vez liberada de las complicaciones de la lucha defensiva, las fuerzas de la sexualidad pregenital son incluidas en la organización genital. Pero lo más importante es el trabajo de elaboración analítica siguiente. Es necesaria la educación del yo para que pueda tolerar derivados cada vez menos patológicos.

Freud dijo una vez que es psicoanálisis todo tratamiento que opera mediante la anulación de la resistencia y la interpretación de la transferencia, es decir, todo método que hace que el yo considere sus conflictos patógenos en su plena significación emocional mediante la anulación de la interpretación de derivados, y especialmente de los derivados, expresados en la transferencia. Este es el único criterio. Que el paciente esté acostado o sentado, que se usen o no determinados rituales en el procedimiento terapéutico, es cosa que poco importa. Para los psicóticos y para los niños así como para ciertos casos caracterológicos, el método «clásico» debe ser modificado. De qué manera se lleva a cabo realmente la labor psicoanalítica, las cuestiones que atañen a la técnica psicoanalítica y a los problemas técnicos especiales, son cosas que ciertamente no caben dentro de una teoría de la neurosis (Fenichel, 1996, p. 635).

El tratamiento psicoanalítico, está indicado en todos los casos en que existen dificultades neuróticas como resultado de un conflicto neurótico. El 
psicoanálisis es una terapia causal de las neurosis. $\mathrm{Su}$ objetivo es hacer que el yo del paciente enfrente lo que anteriormente había rechazado. No se hace un uso inmediato de la transferencia para los fines terapéuticos, sino que se la analiza, es decir, que se le demuestra al paciente el verdadero carácter de la misma. Aquello que anteriormente había sido excluido de la personalidad encuentra nuevamente su vinculación con ella y logra alcanzar una maduración tardía. Las defensas patógenas, en el neurótico, conservan su eficacia gracias a que las angustias, los sentimientos de culpa, y las ideas del mundo y de la vida, creados en cierto momento de la infancia, continúan actuando y se hallan fuera del alcance del yo razonable. Por ello, si no trabajamos las ideas erróneas del mundo y el sentido de la vida en el neurótico de poco sirve el trabajo con las causas a partir de transferencia. Lo removido al tiempo, o ante el primer suceso traumático, se vuelve a instalar. Como afirma Frankl, «...creemos que el tratamiento es inútil mientras no se esclarezca la problemática del sentido de la vida, porque si ésta no se esclarece, la etiología permanece y regresan los síntomas» (Frankl, 1974, p. 339). Por esta razón se destaca la importancia del trabajo integrado a partir de los distintos estratos.

Siguiendo a Lou Marinoff, pionero del asesoramiento filosófico en Estados Unidos de Norteamérica:

Nos encontramos con que ni la ciencia ni la religión pueden responder a todas nuestras preguntas. El psicoterapeuta filósofo Viktor Frankl advirtió que este hecho conducía a un vacío existencial y que la gente corriente necesitaba una nueva vía de salida: cada vez se agolpan más pacientes en nuestras clínicas y consultorios quejándose de vacío interior, de la sensación de una absoluta y definitiva falta de sentido en sus vidas. Podemos definir el vacío existencial como la frustración de lo que cabe considerar la fuerza motivadora más elemental del hombre, a la que podríamos llamar la voluntad de significar. Frankl usó la frase voluntad de significar para emparejar dos de las ideas claves de la psicología: la voluntad de poder de Adler y la voluntad de placer de Freud. Ahora bien, tal como Frankl previó, había algo más profundo en el meollo del problema fundamental de las personas, y los tratamientos médicos, psicológicos y existentes no iban a bastar para aliviarlo (...)

El asesoramiento filosófico tampoco puede aplicarse a todo y es preciso que a veces recomiende asistencia psiquiátrica o psicológica a algunos de mis clientes, como complemento, en sustitución de un análisis filosófico de su situación, o como paso previo a éste. El asesoramiento filosófico es, con mucho, el que ofrece una mayor variedad de enfoques prácticos y duraderos de los problemas más comunes que llevan a las personas a pedir ayuda, y obviamente llena los espacios en blanco que dejan otra clase de asesoramiento (2000, pp. 30-35).

La psicología fracasa cuando está desprovista de un punto de vista filosófico, y el asesoramiento filosófico sólo es eficiente si antes trabajamos lo psicológico profundo. Ambas disciplinas sólo se han empobrecido como resultado de su bifurcación. Fracasan los que pretenden abordar la conflictiva del hombre solo desde lo filosófico; también fracasan las terapias que lo abordan sólo desde lo psicológico o lo biológico. El problema reside en que gran parte de la psicología moderna desciende, o recibe la influencia, de la psicología conductista y su intrínseco empobrecimiento de lo específicamente humano. El sentido de un síntoma no se agota en sus factores biológicos y psicológicos. Hay que añadir, también, el factor noógeno, espiritual o existencial. Se recuerda que el término Psicoterapia procede de dos palabras griegas que no tienen relación alguna con la medicina: Therapeuein, que significa prestar atención a algo, y psykhé que significa alma, aliento o carácter. Por lo tanto, psicoterapia puede significar prestar atención al alma. Una visión dimensional del hombre permite reconocer el modo, también dimensional, de su enfermar. El hombre múltiple en su esencia, necesita abordajes múltiples e integradores para lograr su equilibrio, y, por lo tanto, su salud mental.

\section{CONCLUSIONES}

Para orientar su tarea, el psicólogo necesita tener una idea de hombre. Como decía Goethe: "Si consideramos a un hombre como lo que es, lo veremos peor, si lo consideramos como debería ser, lo podemos 
ayudar a ser lo que podría ser" (Frankl, 1978, p.27) En todas las corrientes psicológicas, existe una idea de persona y de mundo. Pero si se sigue una escuela como letra muerta, el mensaje se endurece. Freud habló de su escuela como construyéndose, como no acabada, y dejó en claro en una carta a Binswanger que él sólo se encargaba de lo instintivo, legándole a este último el trabajo sobre lo espiritual. Frankl completa la idea de hombre de Freud, y gracias al aporte de la Logoterapia, la situación analítica, que parte de la transferencia, puede convertirse en encuentro: la situación neurótica (dimensión psicológica) se desplaza al encuentro analizante-analista (dimensión espiritual), convirtiéndose en una situación dialogal. Desde esta perspectiva, las raíces de todo síntoma neurótico surgen de cuatro dimensiones del ser humano: como producto de algo físico, como expresión de algo psíquico, como manifestación de lo social y como modo de existencia.

El terapeuta sólo podrá lograr que el paciente exprese su filosofía de vida con más claridad, que vea sus aspectos inconscientes, que se haga responsable de su propia vida y sus efectos.

Esto lo llevará a dar una respuesta concreta y creadora al sentido de su existencia; lo ayudará a encarar su vida como una misión trascendente -fuente de felicidad y también de salud psíquica-. Para Aristóteles, la felicidad implica el perfeccionamiento de las potencias específicamente humanas. Es ineludible que, en el ámbito de la psicoterapia, el paciente cuestione sus valores, su modo de ver la vida. Frankl advierte los riesgos de confundir los problemas psicológicos con las cuestiones filosóficas, pero aclara que es necesario el trabajo con ambos. Quien no descubre el sentido de su vida, su vocación de vida, quien no trabaja sus ideas distorsionadas de mundo y de la vida, enferma más fácilmente o vuelve a enfermar.

Si la etiología de la neurosis es multidimensional, la sintomatología es polivalente. Por lo tanto, los modos de abordarlo deben ser diversos, pero complementarios y apuntando a un mismo fin. De esta manera, de concebir al hombre dimensionalmente se pasa a concebir dimensionalmente la enfermedad y también la clínica. ...en tanto se despierta la conciencia del terapeuta, respecto de la unidad, totalidad y dignidad del hombre, cualquier medio terapéutico que se aplique estará a salvo de vulnerar al hombre (...) lo que importa no es la técnica, sino el resumen con que ella es empleada (Fizzotti, 2001, p.181).

Aceptar al hombre en su totalidad es reconocer, en el ámbito de la psicoterapia, no sólo un espacio para trabajar a partir de la transferencia con sus dinámicas intrapsíquicas. Implica, además, trabajar con el resto de su personalidad, con «todo» lo que quedó detenido y reconocer la necesidad de confrontar sobre las cuestiones radicales de la búsqueda de sentido. El trabajo final de la terapia, condición indispensable para el final del tratamiento, es la pregunta por la vocación, por el sentido de la vida, porque, al realizar su genitalidad, las personas realizan su vocación, se realizan a sí mismas. Fizzotti sostiene que la vía que conduce a la felicidad pasa por el coraje de vivir (2001). La psicoterapia debe conducir al reino del valor y del sentido, que en tanto constituyen el reino propio del hombre, son el puerto final de la salud. Se recuerda que los términos salud y salvación provienen del mismo tronco semántico y expresan, en definitiva, una sola realidad.

Frankl no es un sistema, es un universo. La sistemática que se echa de menos, a lo largo de su obra, sin lugar a dudas, tiene un límite, pero reúne la reflexión del espíritu humano de todos los tiempos. Su fascinante novedad reside en la antigüedad de sus formulaciones dándole a la psicología la condición de "paideia" (Caponnetto, 1995, p. 281).

La Logoterapia, escuela complementaria, permite partir del psicoanálisis y, sin olvidar sus limitaciones, trabajar las ideas erróneas del mundo y de la vida en las neurosis de transferencia. Es posible encarar la tarea del psicólogo apelando a la libertad, a la responsabilidad, a la vida vivida como misión trascendente; encarar la psicoterapia como un encuentro dialogal, no como un mero espacio de repetición o de transferencia -aunque algunos de estos aspectos sean utilizados para hacer diagnóstico o para comenzar el tratamiento-. Los aportes de la Logoterapia completan un trabajo que, abordado sólo desde el 
psicoanálisis, en el mejor de los casos, quedaría a mitad de camino. Muchos criticaron a Freud, pero sólo Frankl tuvo la humildad de presentarse como "...el enano en los hombros del gigante" (Ovejas, 2005, p. 246)

\section{REFERENCIAS}

Caponnetto, Héctor (1995). Viktor Frankl. Una Antropología Médica. Buenos Aires: Instituto Bibliográfico Antonio Zinny.

Ey, Henri (1996). Tratado de Psiquiatría. Buenos Aires: Toray-Masson.

Fenichel, Otto (1996). Teoría Psicoanalítica de las Neurosis. Buenos Aires: Paidós.

Fizzotti, Eugenio (2001). Las raíces de la Logoterapia. Buenos Aires: Fundación Argentina de Logoterapia.
Frankl, Viktor (1978). Psicoanálisis y Existencialismo. México: Fondo de Cultura Económica.

Freud, Sigmund (1991). Obras Completas. Buenos Aires: Amorrortu.

Marinoff, Lou (2000). Más Platón y menos Prozac. Barcelona: Ediciones B.

Oro, Oscar (1985). Logoterapia. Buenos Aires: Publicación de Estudios Franklianos y de la Sociedad Argentina de Logoterapia.

Ovejas, Patricia (1992). Repetición y transferencia. Buenos Aires: Hospital Ameghino.

Ovejas, Patricia (2005). Integración de las teorías de Freud y Frankl en el diagnóstico y tratamiento de las neurosis de transferencia. Tesis Doctoral. UCA.

Fecha de recepción: 18 de abril, 2011

Fecha de aceptación: 22 de junio, 2011 